\title{
Psychiatry Workforce and Psychiatry Recruitment: Two Intertwined Challenges
}

\author{
Adam M. Brenner ${ }^{1} \cdot$ Richard Balon ${ }^{2} \cdot$ John H. Coverdale ${ }^{3}$ - Eugene V. Beresin ${ }^{4}$ • \\ Anthony P. S. Guerrero ${ }^{5}$ - Alan K. Louie ${ }^{6} \cdot$ Laura Weiss Roberts $^{6}$
}

Received: 20 January 2017 / Accepted: 25 January 2017 /Published online: 15 February 2017

(C) Academic Psychiatry 2017

The collection of papers on recruitment in the April 2017 issue of Academic Psychiatry provides a wealth of practical wisdom and helps us appreciate that we face two very different problems when considering recruitment into the field of psychiatry. Although at times these problems overlap and certainly are intertwined, we can think of these two problems first as input into the residency system (recruitment) and then as output of the residency system (workforce).

The distinct nature of these problems is most apparent in that although the recruitment rate of US graduates going into psychiatry is fairly low, we nonetheless fill all our general psychiatry programs and go on to graduate these psychiatry residents as part of the physician workforce. Simply increasing recruitment or the percentage of US students who make psychiatry their first choice specialty in the National Resident Matching Program (NRMP) would not automatically address the workforce challenges faced by our field.

Our task is to ask - and begin to answer - two questions: How successful are we in producing cohorts of psychiatrists to meet the population health needs of local communities, the country, and even the world? To accomplish this goal, how

Adam M. Brenner

adam.brenner@utsouthwestern.edu

University of Texas Southwestern Medical Center, Dallas, TX, USA

2 Wayne State University, Detroit, MI, USA

3 Baylor College of Medicine, Houston, TX, USA

4 Harvard Medical School, Boston, MA, USA

5 University of Hawai'i John A. Burns School of Medicine, Honolulu, HI, USA

6 Stanford University, Stanford, CA, USA successful are we in attracting medical students into psychiatry?

The papers in this collection provide excellent perspectives on these questions. In terms of workforce needs, Verduin [1] outlines the problem succinctly. The population of the USA, Verduin notes, has been growing rapidly, but the rate of growth of psychiatrists has not come close to the same pace. Verduin cites the estimate of the Association of American Medical Colleges that within 10 years we will have a deficit of 20,000 to 30,000 psychiatrists [2]. One way of understanding the workforce shortage is to recognize that in the past 20 years we have seen approximately a $37 \%$ increase in population with only a $12 \%$ increase in number of psychiatrists (interestingly, the number of physicians overall has increased by $45 \%$ ) [3]. Another aspect of the shortage is that the total number of psychiatrists does not provide the whole picture when it comes to access to care because it can be far more difficult for some patients to find psychiatrists who are providers for specific insurance plans, particularly Medicaid [4].

When considering workforce, we need to not only appreciate the growth of the population but also consider the population's current unmet psychiatric needs. The lifetime prevalence of psychiatric disorders worldwide is approximately $24 \%$ [5]. The fact that almost one in four individuals will experience a psychiatric disorder requires an additional look at our workforce, in terms of not only overall graduates but also our training of primary care physicians and allied health professionals and our collaboration with them in a wide range of settings. The current situation is particularly acute for the mental health needs of children, adolescents, and transitional youth. Estimates indicate that $50 \%$ of psychiatric disorders begin before age 14 and about $75 \%$ begin in transitional age youth (ages 14-26) and that 14-20 million youth have severe psychiatric disorders. We currently have approximately 8000 child and adolescent psychiatrists across the entire country to 
meet the needs of our population. This workforce shortage results in undertreatment of youth, and if psychiatry is also mandated to work toward prevention and early intervention, we fall far short of the mark needed to provide optimal care.

Similar deficits in workforce have been described in the United Kingdom, and there are even more severe shortages in developing countries [6]. As Harper and Roman [7] note, the increase of psychiatry slots in the match (31 more in 2016 than 2015) will come nowhere close to filling the gap in the USA. As Walaszek [8] notes, the aging of the population is inevitably further increasing the demand for geriatric expertise, which is unlikely to be met by the output of geriatric fellowships, even if a greater proportion of the psychiatry cohort select to train in geriatric psychiatry. In other words, we are at a greater psychiatrist deficit than the raw numbers likely indicate for certain populations.

Some academic psychiatrists may find it hard to square these realities with their experiences because many of us work in major metropolitan areas where the workforce problem is less in evidence. Because most psychiatrists are trained in major cities, cities tend to be where most graduates find their initial employment. Although geographic spread may occur over the years, we are left with staggering disparities of psychiatrist availability in urban versus rural environments. Thomas et al. [9] found that around $75 \%$ of counties across the USA have a shortage of prescribing mental health clinicians, including most prominently psychiatrists. For example, in Texas (home to two of the authors, AB and JC), $10 \%$ of the population lives in counties that have no psychiatrists whatsoever. Sixty-three percent of all psychiatrists in the state work in the five most populous counties, leaving the remaining 207 counties with a ratio of 21,000 citizens per psychiatrist; conservative estimates set an adequate ratio at approximately 4000 people per psychiatrist [9]. Other states, like Hawai'i (home to one of the authors, AG), have obvious geographical barriers that further isolate populations away from available psychiatrists.

The workforce problem is compounded further by the "graying" of physicians, including psychiatrists, and a recognition that a sizeable proportion of the workforce will soon retire [10]. Walaszek [8] notes that psychiatry is now the third oldest specialty in the USA. A 2013 Substance Abuse and Mental Health Services Administration [11] report found that the median age of psychiatrists was 55.7 years, and most troubling, $46 \%$ were over 65 years old. We are fortunate that we are in a specialty where clinicians do not "peak" young, and they may continue to develop their knowledge and clinical wisdom in their $50 \mathrm{~s}, 60 \mathrm{~s}$, or beyond. Nevertheless, it is not realistic to expect that most older psychiatrists will continue to practice for many decades to fill in gaps in service.

Now, let us turn to the question of recruitment. From the NRMP data, the percentage of matched positions that went to psychiatry has been roughly consistent at $5 \%$ over past years.
Since the total number of matched positions has increased, so too have the number of positions in psychiatry, from 1117 in 2012 to 1384 in 2016.

How did our field do in filling these positions? Overall, very well. In 2016, 99.2\% of positions (1373) were filled in the match. Only $61.4 \%$ of positions were filled by graduating US medical students, however. As Verduin [1], Harper and Roman [7], and Walaszek [8] describe, numerous reasons likely contribute to this situation in which many slots are filled by physicians who did not graduate from US medical schools. The admissions criteria at many medical schools may not be favorable to individuals interested in psychiatry. Not only may we be skewing our sample of future physicians through the admissions process but we also may need to appreciate that considerable stigma against psychiatry remains within the medical community [12]. Despite increasing recognition of the mental health needs of children and adults, many medical students are steered away from psychiatry. This bias is both overt and covert.

The total percentage of US psychiatry residency positions filled has trended up over the past 5 years, from $96.7 \%$ in 2012. The percentage filled by fourth-year US medical students has varied over the past 5 years, ranging between a low of $51.8 \%$ in 2014 and a high of $61.4 \%$ in 2016 . The trend has been steadily upward, however, when considering the percentage of fourth-year US medical students who chose to match into psychiatry in the past 5 years, from $3.9 \%$ in 2012 to $5.0 \%$ in 2016. This rate of recruitment of fourth-year US medical students to psychiatry is still, however, much lower than the rate of $10 \%$ that occurred in the $1960 \mathrm{~s}$.

Is the relatively low rate of recruitment of US medical graduates to psychiatry a problem if we are succeeding in filling the remaining positions with qualified international medical graduate (IMG) physicians? The argument has been made that IMG physicians fill a crucial role, because they are more likely to provide care for patients in the public sector and therefore in underserved populations, an important area of mental health care disparity $[13,14]$. Unfortunately, invoking this benefit of IMG physician recruitment may only amount to robbing Peter to pay Paul when we broaden our perspective to global psychiatry. Katschnig [6] argues that we may be unintentionally supporting a brain drain from developing countries, citing a World Psychiatric Association Task Force report that raises concern that when deficits of psychiatrists in industrialized countries are filled by international medical graduates, the severe deficits of psychiatrists in less developed nations become even worse.

Aside from the fundamental numbers, how well are we doing in competing for the top US medical students that we would most like to recruit? This is a very difficult question to answer. If we look at academic credentials such as the United States Medical Licensing Examination scores, Alpha Omega Alpha Honor Medical Society membership, and number of 
publications, students who eventually matched into psychiatry were below the national mean in each category [15]. If such categories define the "best and the brightest," clearly we have work to do. Many would argue, however, that these metrics are poor reflections of aptitude for psychiatry and also may reflect stigma toward our specialty of medicine. Walaszek [8] wisely suggests that membership in the Gold Humanism Honor Society may be a more accurate indicator of the qualities that make an effective psychiatrist. At this time, data are lacking regarding how we compare to other specialties with attracting these students. Nevertheless, none of these potential indicators have been proven to predict outcomes for psychiatrists related to clinical performance. We need to define what "the best and brightest" really means for our specialty and study the predictive value of the mentioned indicators in an organized retrospective or prospective fashion.

Goldenberg and Krystal [16] provide an important service in analyzing how well we are succeeding in attracting undergraduate neuroscience majors among medical students. This group would appear to include students with interests that align with our field who may eventually assist with further integrating neuroscience into our training [17]. Unfortunately, Goldenberg and Krystal [16] noted that we struggle to attract these students, with only $5.1 \%$ having selected psychiatry at graduation. This percentage is roughly the same as our recruitment rate among the general population of US medical students. By contrast, psychology majors who become medical students are three times more likely than their peers to choose psychiatry. It remains to be seen whether the growing pool of premedical students with neuroscience majors includes a significant number with an aptitude and interest in treating patients with mental illness. And it remains to be studied if students entering medical school with an interest in psychiatry are driven away due to stigma, hidden curricula that diminish the value of psychiatry, and/or by the low salaries that may be expected in practice as a psychiatrist.

To develop a workforce resembling the wider society, psychiatry must recruit students from racial and ethnic minority groups, students whose parents have not attended college, and students who have come from impoverished and low-income backgrounds. The presumption is that psychiatrists with these backgrounds are underrepresented in the workforce. Moreover, these personal histories and identities should promote a diversity of ideas and assist in reducing disparities and addressing important public health problems [18]. Anecdotally, many underrepresented minority medical students who consider psychiatry maintain a strong interest in community psychiatry and global mental health.

Having established that we have work to do in meeting the challenges of workforce needs and recruitment, what strategies are available to us, and how do the interventions potentially overlap? Potential interventions can be grouped into three types - those that address improving recruitment, those that address workforce deficits, and those that potentially address both.

The great deal of convergence in the recruitment recommendations of Verduin [1], Walaszek [8], and Harper and Roman [7] provides evidence for an expert consensus regarding attracting students to psychiatry. This consensus approach would include the following:

- advocating on admissions committees for the selection of such students who might be inclined toward psychiatry,

- identifying interested students early in medical school and offering quality enrichment programs and active mentoring,

- ensuring clerkship length and quality comparable to other major specialties and possibly introducing longitudinal clerkships to better demonstrate the effectiveness of psychiatric treatments,

- exposing students to faculty who are charismatic teachers and who demonstrate that psychiatry is a fulfilling career that supports the wellness of the clinician,

- actively rebutting the persistent stigma of a career in psychiatry, and

- revising medical school curricula to integrate psychiatric and psycho-social issues as crucial parts of the practice of medicine.

Walaszek [8] and Verduin [1], furthermore, independently echo the recommendation of Goldenberg and Krystal [16] that there may be untapped recruitment opportunities at the undergraduate (collegiate) level. Academic psychiatrists might consider seeking out teaching positions in undergraduate neuroscience, psychology, and the humanities to clearly illustrate real-world applications of clinical interests and public health priorities.

Because we are currently filling virtually all psychiatry residency positions, the psychiatry workforce shortage clearly is not an issue that can be adequately addressed simply by recruitment efforts. Approximately 49,000 to 50,000 psychiatrists are currently in practice in the USA. If we assume that an average psychiatrist will work 40 years after residency training, the presently available number of residency slotsapproximately 1400 - will only allow for the replacement of the existing force. This formula does not consider that some people, especially those involved in taking care of children or older relatives, may not work full time or that some will retire earlier than at age 70 (if psychiatrists routinely retired at age 65 , we would not even be able to replace the existing workforce).

The most obvious solution for addressing workforce deficits - increasing the number of psychiatry residency slots-is, of course, limited by available funding. An increase, especially in the current political climate, may be impossible. Academic psychiatrists have an important role to play in joining with professional organizations such as the American 
Psychiatric Association in advocating on behalf of our field and our patients for increased residency funding. Although no one can predict with certainty, it seems unlikely that we will see a significant increase in federal funding for graduate medical education through Medicare in the near future. Nonetheless, other options remain. The Veteran's Administration has made funding available in recent years for an expansion of residency positions at some of their hospitals. State legislatures can also at times be convinced of the need to act when workforce shortages become apparent locally. Residency educators ideally will partner with health policy institutions and experts and with major mental health employers to establish a body of evidence that demonstrates regional hiring difficulty. State legislative bodies can then be made aware that failing to fund an adequate workforce in psychiatry is simply poor fiscal management, because the result is a greater accumulation of expense due to patients with serious mental illness in crisis care and in the correctional/ justice systems. This kind of advocacy should not be viewed as a "guild" issue. We need to remind our leaders that a $24 \%$ lifetime prevalence of mental illness is not insignificant, that we are in a national crisis in addiction, that suicide is the second leading cause of death in our transitional age youth, and that depression is the leading cause of the global burden of illness. Without an adequate psychiatry workforce, we will continue to drain our health care system and society of productive individuals and add to the economic burden of psychiatric illness.

We may also potentially address workforce deficits by more effectively leveraging and deploying our current number of graduates. This effort could mean, as Walaszek [8] describes, increasing our training of general psychiatry graduates in areas such as addiction and geriatrics, and even child and adolescent care, so that a higher percentage of general psychiatrists are available for the treatment of these growing populations. We could focus on multiplying the individual psychiatrist's impact by emphasizing and standardizing training that prepares psychiatry graduates to consult with primary care doctors and to supervise other mental health practitioners. Given the likelihood of persistent shortages, beyond our collaborating with other clinicians in medical homes and accountable care organizations, we need to teach our current and future psychiatrists to harness innovation, for example, telepsychiatry, to a far greater extent for purposes of consultation/liaison, evaluation, and treatment. Doing so, of course, may require opening our ability to practice psychiatry across state lines through licensure and reimbursement from insurance companies to provide improved access to care, not simply for consultation but also for ongoing treatment.

Finally, potential interventions may have an impact on both recruitment and the workforce. Verduin [1] and Harper and Roman [7] discuss the potentially chilling impact that medical student debt may have on the choice of psychiatry, which is a relatively low-paying specialty option. Verduin [1] notes that the average debt of medical school graduates is approximately $\$ 180,000$. Debt relief programs may therefore help with recruiting, and these programs can be made contingent on residency graduates working in areas of our field where the workforce shortage is most acute. Currently, programs in some states offer debt relief to psychiatry graduates who work in the public sector. It may be wise to consider developing similar programs to encourage service for specialty geriatric, addiction, and child psychiatry patients, all of whom face shortages that are likely to be even more severe than for general psychiatry patients [19].

The collection of papers in the April 2017 issue of Academic Psychiatry offer a valuable analysis of obstacles and opportunities in psychiatry recruitment and provide many examples of best practices for medical student and residency educators. The authors help us appreciate that we cannot approach recruitment rationally without taking into account workforce needs and trends in population mental health. As we have seen, these two matters, recruitment and workforce, are separate strands that must be woven together to provide a stronger bridge into our field for the sake of our patients and our potential patients.

\section{Compliance with Ethical Standards}

Disclosures On behalf of all authors, the corresponding author states that there is no conflict of interest.

\section{References}

1. Verduin ML. Improving the recruitment of medical students into psychiatry. Acad Psychiatry. 2017. doi:10.1007/s40596-016-0651-7.

2. Mann S. AAMC research confirms looming physician shortage. AAMC Reporter, April 2016. Available at https://news.aamc.org/ medical-education/article/aamc-research-physician-shortage/. Last accessed 19 January 2017.

3. Sederer L. Where have all the psychiatrists gone? Doctors are struggling to meet the growing demand for psychiatric help across the U.S. US News and World Report, September 15, 2015. Available at http://www.usnews.com/opinion/blogs/policy-dose/2015/09/15/ the-us-needs-more-psychiatrists-to-meet-mental-health-demands. Last accessed 19 January 2017.

4. Wilk JE, West JC, Narrow WE, Rae DS, Regier DA. Access to psychiatrists in the public sector and in managed health plans. Psychiatr Serv. 2005;56:408-10.

5. World Health Organization. The World Health Report 2001. Mental health: new understanding, new hope. Available at http://www. who.int/whr/2001/en/whr01_en.pdf?ua=1. Last accessed 19 January 2017.

6. Katschnig H. Are psychiatrists an endangered species? Observations on internal and external challenges to the profession. World Psychiatry. 2010;9:21-8.

7. Harper BL, Roman B. The changing landscape of recruitment in psychiatry. Acad Psychiatry. 2017. 
8. Walaszek A. Keep calm and recruit on: residency recruitment in an era of increased anxiety about the future of psychiatry. Acad Psychiatry. 2017.

9. Thomas KC, Ellis AR, Konrad TR, Holzer CE, Morrissey JP. County-level estimates of mental health professional shortage in the United States. Psychiatr Serv. 2009;60:1323-8.

10. Kupfer JM. The graying of US physicians. Implications for quality and the future supply of physicians. JAMA. 2016;315:341-2.

11. U.S. Department of Health and Human Services. Substance Abuse and Mental Health Services Administration. Report to Congress on the Nation's Substance Abuse and Mental Health Workforce Issues. January 24, 2013. Available at https://store.samhsa.gov/shin/ content/PEP13-RTC-BHWORK/PEP13-RTC-BHWORK.pdf. Last accessed 19 January 2017.

12. Schlozman S, Beresin EV, Balon R, et al. Stigma and mental health: a proposal for next steps. Acad Psychiatry. 2016;40:735-9.

13. Pierre JM, Mahr F, Carter A, Madaan V. Underrepresented in medicine recruitment: rationale, challenges, and strategies for increasing diversity in psychiatry residency programs. Acad Psychiatry. 2016. doi:10.1007/s40596-016-0499-x.
14. Rao NR. Recent trends in psychiatry residency workforce with special reference to international medical graduates. Acad Psychiatry. 2003;27:269-76.

15. National Resident Matching Program. Charting outcomes in the match for U.S. allopathic seniors: characteristics of U.S. allopathic seniors who matched to their preferred specialty in the 2016 main residency match. Available at http://www.nrmp.org/wp-content/ uploads/2016/09/Charting-Outcomes-US-Allopathic-Seniors2016.pdf. Last accessed 19 January 2017.

16. Goldenberg M, Krystal J. Undergraduate neuroscience majors: a missed opportunity for psychiatry workforce development. Acad Psychiatry. 2017.

17. Ross DA, Travis MJ, Arbuckle MR. The future of psychiatry as clinical neuroscience: why not now? JAMA Psychiatry. 2015;72: 413-4.

18. Roberts LW, Maldonado Y, Coverdale J, Balon R, Louie AK, Beresin EV. The critical need to diversify the clinical and academic workforce. Acad Psychiatry. 2014;38:394-7.

19. Vernon DJ, Salsberg E, Erikson C, Kirch DG. Planning the future mental health workforce: with progress on coverage, what role will psychiatrists play? Acad Psychiatry. 2009;33:187-92. 